\title{
Combination Therapy of Metastatic Castration-Recurrent Prostate Cancer: Hyaluronic Acid Decorated, Cabazitaxel-Prodrug and Orlistat Co-Loaded Nano-System
}

\section{Zhen Qu \\ Yuning Ren \\ Hongyu Shen \\ Huihui Wang \\ Lijie Shi \\ Deyong Tong}

Department of Oncology, 970 Hospital of the PLA Joint Logistic Support Force, Yantai, 26400I, People's Republic of China
Correspondence: Deyong Tong Department of Oncology, 970 Hospital of the PLA Joint Logistic Support Force, No. 7, Zhichu South Road, Zhifu District, Yantai City, Shandong Province, People's Republic of China

Email tongdypla@tom.com
Purpose: Prostate cancer ( $\mathrm{PCa}$ ) is the second leading cause of cancer-related death among men in developed countries. Cabazitaxel (CBZ) is recommended as one of the most active chemotherapy agents for PCa. This study aimed to develop a hyaluronic acid (HA) decorated, cabazitaxel-prodrug (HA-CBZ) and orlistat (ORL) co-loaded nano-system against the prostate cancer in vitro and in vivo.

Methods: Cabazitaxel-prodrug was firstly synthesized by conjugating HA with CBZ through the formation of ester bonds. HA contained ORL and CBZ prodrug co-loaded lipidpolymer hybrid nanoparticles (ORL/HA-CBZ/LPNs) were constructed and characterized in terms of particle size, zeta potential, drug loading capacity and stability. The antitumor efficiency and systemic toxicity of LPNs were evaluated in vitro and in vivo.

Results: The resulting ORL/HA-CBZ/LPNs were $150.9 \mathrm{~nm}$ in particle size with narrow distribution and high entrapment efficiency. The minimum combination index of 0.57 was found at a drug ratio of 1:2 (ORL:HA-CBZ, w/w) in the drug co-loaded formulations, indicating the strongest synergism effect. ORL/HA-CBZ/LPNs demonstrated an enhanced in vitro and in vivo antitumor effect compared with single drug loaded LPNs and free drug formulations.

Conclusion: ORL/HA-CBZ/LPNs showed remarkable synergism cytotoxicity and the best tumor inhibition efficiency in mice with negligible systemic toxicity. ORL/HA-CBZ/LPNs can be highly useful for targeted prostate cancer therapy.

Keywords: metastatic castration-recurrent prostate cancer, cabazitaxel, prodrug technology, orlistat, hyaluronic acid

\section{Introduction}

Prostate cancer (PCa) is the second leading cause of cancer-related death among men in developed countries. ${ }^{1,2}$ According to the NCCN guideline, it has been recommended that androgen deprivation therapy (ADT) is the gold standard therapy for patients with early-stage, low-risk disease and advanced or metastatic disease. $^{3,4}$ As the disease progresses, the majority of advanced PCa can evolve into metastatic castration-recurrent $\mathrm{PCa}$ (mCRPC). For patients with mCRPC, docetaxel is a standard regimen. However, taxane (docetaxel) resistance hinders its efficacy; thus, cabazitaxel, abiraterone acetate or enzalutamide are recommended as an alternative therapy. 
Cabazitaxel (CBZ), a second-generation taxane, has been approved by the FDA for men with metastatic CRPC previously treated with a docetaxel-containing regimen since June 2010, and is considered one of the most active chemotherapy agents. ${ }^{5,6} \mathrm{CBZ}$ is able to overcome the drug efflux pump P-glycoprotein (Pgp)mediated transport, which leads to docetaxel resistance. $^{7}$ Consequently, CBZ was 10 -fold more potent than docetaxel in chemotherapy-resistant tumor cells. ${ }^{8}$ Jevtana, the commercialized CBZ formulation, is a common injection, containing polysorbate 80 and diluents $(13 \% \mathrm{w} / \mathrm{w}$ ethanol in water) for surmounting its poor water solubility. Its solubilizing formulation causes serious side effects and has no selectivity between healthy tissues and tumor tissues. ${ }^{9,10}$ Meanwhile, its overall median survival benefit is 2.4 months. ${ }^{11}$ Therefore, it is urgent to exploit new strategies to improve its target as well as therapeutic efficacy, and decrease its side effects.

Prodrug technology has been applied to enhance its solubility, its tumor target and efficacy. ${ }^{12}$ Several researchers have taken advantage of poly(ethylene glycol) PEGbased prodrug to synthesize cabazitaxel-PEG prodrugs that are capable of accumulating in prostate tumor tissues through passive targeting, the enhanced permeability and retention (EPR) effect. ${ }^{13,14}$ Compared with passive targeting, prodrug-based active targeting (cancer cell-specific targeting) has attracted wide attention in cancer therapy. ${ }^{15}$ Hyaluronic acid (HA), a glycosaminoglycan polymer, has the capability to bind to HA principal cell surface receptor, CD44, that is a multifunctional cell surface glycoprotein involved in cell proliferation and cell migration. ${ }^{16}$ Various studies have identified that CD44 expression is higher in PCa tissues than in normal tissues, and different kinds of $\mathrm{HA}$ targeted nanoparticles were engineered for higher PCa therapeutic efficacy. ${ }^{17-19}$ In this study, a novel active targeting CBZ prodrug was synthesized by the hydrolysable ester bond between cabazitaxel (the hydrophobic part) and hyaluronic acid (the hydrophilic part).

Combination therapy is another strategy that can be used to conquer drug resistance, improve anticancer efficacy, and reduce side effects. Orlistat (ORL), an effective fatty acid synthase (FASN), has been researched to enhance taxane (paclitaxel, docetaxel and cabazitaxel) sensitivity in resistant cancer cells, including prostate cancer cells, breast cancer cells and hepatocellular carcinoma cell lines. ${ }^{20-24}$ ORL is a hydrophobic drug, and nanotechnology is applied in our study to surpass this shortcoming and co-deliver ORL and CBZ prodrug.

Self-assembled amphiphilic prodrug-based nanosystems could overcome the limitations of common prodrugs, which on their own may be degraded in vivo in an uncontrolled manner. ${ }^{25}$ Nano-systems were also reported to have the ability to improve the therapeutic efficacy and reduce the systemic toxicity by enhancing the pharmacokinetics parameters, such as large volume distribution and tumor deposition of the payloads through enhanced permeability and retention (EPR) effect. ${ }^{26}$ Among all kinds of nano-systems, lipid-polymer hybrid nanoparticles (LPNs) combine the advantages of polymeric and lipid nanoparticles into one novel drug delivery platform. ${ }^{27}$ The present study describes an LPNs system co-delivery of ORL and CBN prodrugs.

In the present study, HA contained CBZ prodrug and ORL co-loaded LPNs (ORL/HA-CBZ/LPNs) were constructed and characterized in terms of particle size, zeta potential, drug loading capacity and stability. The antitumor efficiency and systemic toxicity of NPs were evaluated in vitro and in vivo.

\section{Materials and Methods Materials}

Hyaluronic acid was obtained from Freda Biochem Co., Ltd. (Ji'nan, China). Poly (D,L-lactic-co-glycolic acid) (PLGA), soybean lecithin (SL), 1,2-distearoyl-sn-glycero-3-phosphoethanolamine-N-carboxy (polyethylene glycol) 2000 (DSPE-PEG), CBZ, ORL, 1-ethyl-3-(3-dimethylaminopropyl) carbodiimide (EDC), N-hydroxysuccinimide (NHS), 3-[4, 5-dimethylthiazol-2yl]-2, 5 diphenyltetrazolium (MTT), and coumarin-6 (C6) were obtained from Sigma Aldrich (St. Louis, MO).

\section{Synthesis of Cabazitaxel-Prodrug}

Cabazitaxel-prodrug (HA-CBZ) was synthesized by conjugating $\mathrm{HA}-\mathrm{COOH}$ with $\mathrm{CBZ}-\mathrm{OH}$ through the formation of ester bonds (Figure 1). HA-COOH (2 equivalents), EDC $\cdot \mathrm{HCl}$ (4 equivalents), NHS (4 equivalents), DMAP (2 equivalents) were dissolved in acetonitrile. ${ }^{28,29} \mathrm{CBZ}$ $\mathrm{OH}$ (1 equivalent) was dissolved in dimethylformamide and added to the above HA containing acetonitrile solution. When the mixture was stirred overnight, the solid precipitates in the reaction mixture were removed through filtration. HA-CBZ was purified by column 


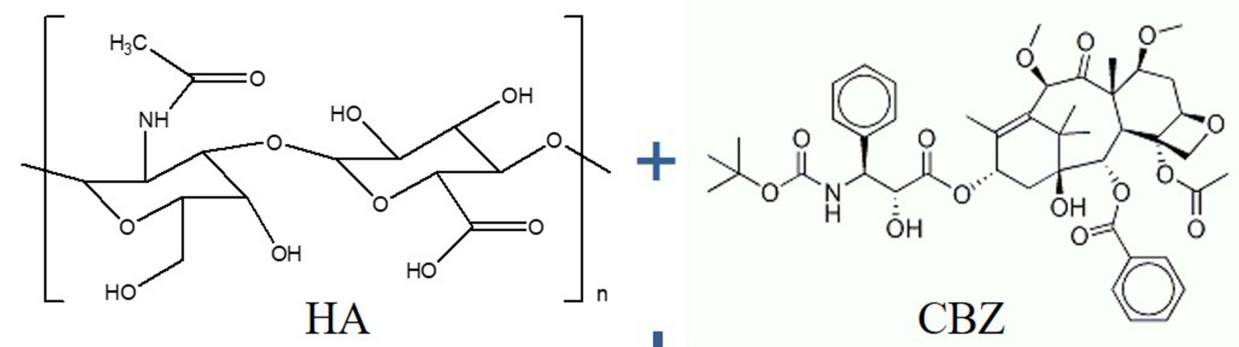

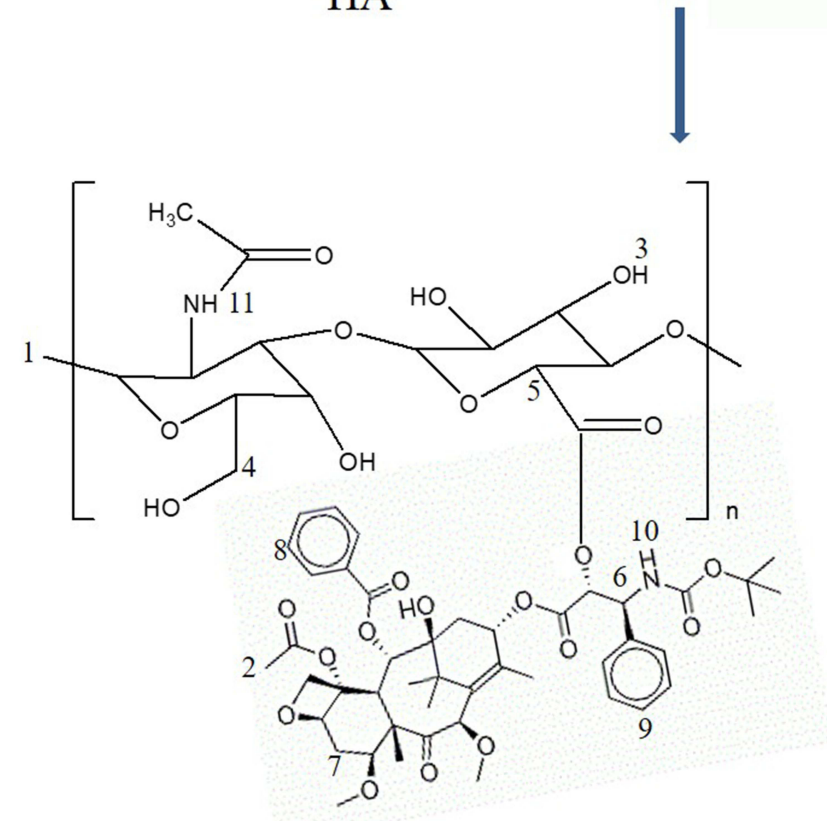

$\mathrm{HA}-\mathrm{CBZ}$

Figure I Synthesis and the 'H NMR spectrum of cabazitaxel-prodrug (HA-CBZ).

chromatography, vacuum drying and analyzed by ${ }^{1} \mathrm{H}-\mathrm{NMR}$ spectroscopy.

\section{LPNs Preparation}

LPNs were self-assembled via a single-step nanoprecipitation method. ${ }^{14}$ Briefly, $200 \mathrm{mg}$ of PLGA polymer, $20 \mathrm{mg}$ of ORL and $40 \mathrm{mg}$ of HA-CBZ were dissolved in $20 \mathrm{~mL}$ of acetonitrile (solution A). $50 \mathrm{mg}$ of SL and $50 \mathrm{mg}$ of DSPE-PEG were dissolved in an ethanol aqueous solution $(4 \%, v / v)$, heated to $70^{\circ} \mathrm{C}$ (solution B). Solution A was then added to solution $\mathrm{B}$ dropwise under gentle stirring for $2 \mathrm{~h}$ at room temperature. Organic solvents and free molecules were removed by washing the solution three times using an Amicon Ultra-4 centrifugal filter (weight cut-off of $10,000 \mathrm{Da})$ to obtain ORL/HA-CBZ/LPNs. HA contained, no drug loaded LPNs (HA-LPNs) were prepared using HA instead of HA-CBZ. Single drug loaded LPNs (ORL/LPNs and HA-CBZ/LPNs) and blank LPNs were prepared using a single ORL (20 mg), HA-CBZ (40 mg) or no drug.

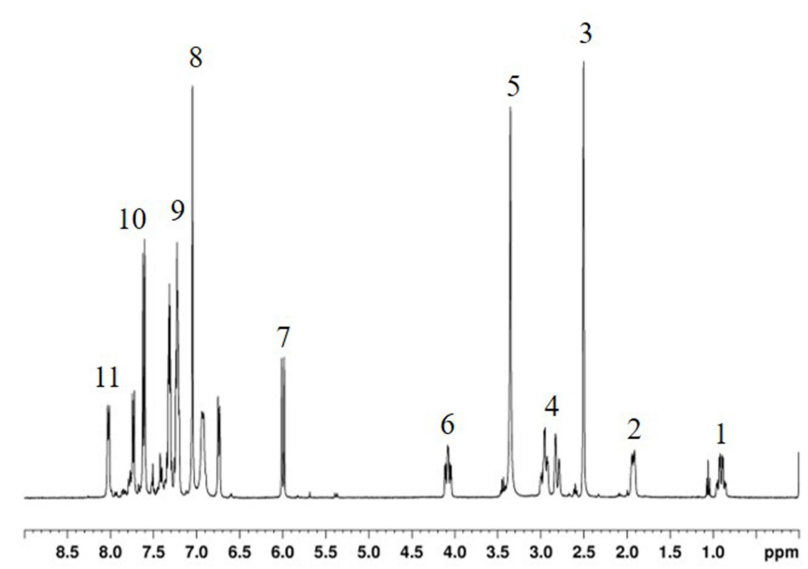

\section{Particle Size, Zeta Potential and Morphology Analysis}

The particle size (mean diameters), polydispersity index (PDI), and zeta potential of LPNs were measured by photon correlation spectroscopy (PCS) using a Zetasizer Nano ZS (Malvern Instruments, UK). ${ }^{30}$ Briefly, LPNs were diluted at $2 \%(\mathrm{v} / \mathrm{v})$ in $1 \mathrm{mM} \mathrm{NaCl}$ in triplicate, and three independent measurements were performed in disposable folded capillary cells with a $173^{\circ}$ scattering angle at $25^{\circ} \mathrm{C} .^{31}$ To characterize the morphology of the ORL/ HA-CBZ/LPNs, they were imaged by transmission electron microscopy (TEM).

\section{Stability of LPNs}

Serum stability of LPNs was determined by the changes in the mean diameter of the LPNs. ${ }^{32}$ Firstly, LPNs were incubated in PBS ( $\mathrm{pH} 7.4$ ) containing 50\% FBS at $37{ }^{\circ} \mathrm{C}$. In addition, the LPNs long-term stability was determined by storing the LPNs at $4{ }^{\circ} \mathrm{C}$. The particle 
size was measured by the same method in "Particle size and zeta potential analysis" section.

\section{Entrapment Efficiency and Drug Loading}

The entrapment efficiency (EE) and drug loading (DL) of LPNs were evaluated by HPLC method using a reverse column with $\mathrm{C} 18,5 \mu \mathrm{m}, 4.5 \mathrm{~mm} \times 25 \mathrm{~cm} .{ }^{33}$ The HPLC analysis was performed with a flow rate of $1.5 \mathrm{~mL} / \mathrm{min}$. The injected volume of the sample was $10 \mu \mathrm{L}$, and UV detection was monitored at $195 \mathrm{~nm}$ for ORL. ${ }^{34}$ For CBZ testing, the detection wavelength was $230 \mathrm{~nm} .{ }^{35}$ The EE and DL were calculated using the equations:

$\mathrm{EE} \%=($ Total amount of drug added to the system - the amount of free drug in the system)/Total amount of drug added to the system $\times 100$;

$\mathrm{DL} \%=($ Total amount of drug added to the system

- the amount of free drug in the system)/Total amount of carrier material in the system $\times 100$.

\section{In vitro Drug Release of LPNs}

In vitro drug release of LPNs was evaluated using a dialysis method. ${ }^{36}$ Drugs loaded LPNs were sealed in dialysis bags (weight cut-off of $3500 \mathrm{Da}$ ) placed on PBS at $\mathrm{pH} 7.4$ under $100 \mathrm{rpm}$ and constantly shaken. The release medium $(0.5 \mathrm{~mL})$ was taken out at determined time intervals, and the amount of released drugs was calculated by the same method in "Entrapment efficiency and drug loading" section.

\section{Cell Culture and Mice Xenograft}

The human prostate cancer cell lines ( $\mathrm{LNCaP}$ and PC3 cells that express $\mathrm{CD} 44^{37,38}$ ) and human prostate normal cells (RWPE-2 cells) were received from the American Type Culture Collection (ATCC, Manassas, VA, USA) and cultured in DMEM containing $10 \%$ heat-inactivated fetal bovine serum, $100 \mathrm{U} / \mathrm{mL}$ penicillin, and $0.1 \mathrm{mg} / \mathrm{mL}$ streptomycin at $37^{\circ} \mathrm{C}$ in a humidified $5 \% \mathrm{CO}_{2}$ incubator.

Male BALB/c nude mice (4-6-week-old) were obtained from Beijing Vital River Laboratory Animal Technology Co., Ltd (Beijing, China) and $\mathrm{PCa}$ xenograft mice were generated by injecting $\mathrm{LNCaP}$ cells $\left(1 \times 10^{7}\right.$ each) into the abdominal cavity of mice. The mice are maintained and treated in compliance with the policy of the National Institutes of Health guide for the care and use of laboratory animals and the animal experiments were approved by the Institutional Animal Care and Use Committee of the 970 Hospital of the PLA Joint Logistic Support Force.

\section{In vitro Anti-Proliferative Effect of LPNs}

In vitro anti-proliferative effect of LPNs was assessed using MTT assay to determine the cell viability. ${ }^{39}$ Various concentrations of LPNs and free drugs (from 0.1 to $100 \mu \mathrm{M}$ ) were treated to LNCaP, PC3 or RWPE-2 cells $\left(5 \times 10^{3}\right.$ cells/well $)$ and incubated for $48 \mathrm{~h}$. Then, the medium was removed and added $20 \mu \mathrm{L}$ of MTT solution $(5 \mathrm{mg} / \mathrm{mL})$ into each well, incubated for another 4 h. After the removal of unreduced MTT and old culture medium, $150 \mu \mathrm{L}$ of DMSO was added to each well to dissolve the formazan crystals. The plate was shaken for 10 minutes and the absorbance was read at $570 \mathrm{~nm}$ and the $50 \%$ growth inhibition $\left(\mathrm{IC}_{50}\right)$ was calculated.

\section{Synergistic Effects of LPNs}

Combination index (CI) analysis was applied using Chou and Talalay method to evaluate the synergistic effects of ORL and HA-CBZ in one LPNs system on LNCaP cells. ${ }^{41}$ Combination Index, when $50 \%$ growth inhibition was achieved $\left(\mathrm{CI}_{50}\right)$, values of ORL/HA-CBZ/LPNs were calculated by the equation: ${ }^{42}$

$\mathrm{CI}_{50}=\left(\mathrm{IC}_{50}\right)_{\mathrm{ORL}} /(\mathrm{C})_{\mathrm{ORL}}+\left(\mathrm{IC}_{50}\right)_{\mathrm{HA}-\mathrm{CBZ}} /(\mathrm{C})_{\mathrm{HA}-\mathrm{CBZ}}$, where $\left(\mathrm{IC}_{50}\right)_{\mathrm{ORL}}$ and $\left(\mathrm{IC}_{50}\right)_{\mathrm{CBZ}}$ represent the $\mathrm{IC}_{50}$ values of ORL and CBZ in the single drug loaded LPNs, while $(\mathrm{C})_{\mathrm{ORL}}$ and $(\mathrm{C})_{\mathrm{CBZ}}$ represent the concentration of ORL and $\mathrm{CBZ}$ in the ORL/HA-CBZ/LPNs combination system at the $\mathrm{IC}_{50}$ value. $\mathrm{CI}>1$ represents antagonism, $\mathrm{CI}=1$ represents additive and $\mathrm{CI}<1$ represents synergism.

\section{Cellular Uptake of LPNs}

Cellular uptake of LPNs was analyzed using coumarin-6 (C6) as an indicator. ${ }^{26} \mathrm{C} 6$ loaded LPNs were prepared by the method in section 2.3 adding C6 $(20 \mathrm{mg})$ along with the PLGA to get solution A. LNCaP cells were seeded at on 24-well black plates. C6 loaded LPNs were added to replace the culture medium and incubated with the cells for $4 \mathrm{~h}$. Then, the cells were washed three times with D-Hank's solution, collected and centrifugated. Cell uptake efficiency was quantified using a BD FACSCalibur flow cytometer.

\section{In vivo Tissue Distribution and Pharmacokinetics of LPNs}

When the tumor volume of $\mathrm{PCa}$ xenograft mice reached about $100 \mathrm{~mm}^{3}$, the mice were randomly divided into four groups (10 mice per group): ORL/HA-CBZ/LPNs, HA-CBZ /LPNs, ORL/LPNs, and free ORL/HA-CBZ were injected 
via the tail vein at certain drug doses (ORL $5 \mathrm{mg} / \mathrm{kg}$ and/or HA-CBZ $10 \mathrm{mg} / \mathrm{kg}) .{ }^{39,43}$ The blood samples of mice were taken through tail vein puncture and were centrifuged (3400 g, 15 min at $4{ }^{\circ} \mathrm{C}$ ), and the supernatant plasma was collected and stored at $-80^{\circ} \mathrm{C}$. Mice were euthanized at $24 \mathrm{~h}$, tissues including the tumor, heart, liver, spleen, lung, and kidney were excised. The tissue samples were rinsed with saline, weighed, and stored at $-80^{\circ} \mathrm{C}$. The tissues were homogenized in lysis buffer, mixed with methanol, and then centrifuged for $30 \mathrm{~min}$, then analyzed along with the blood samples were by the same method in "Entrapment efficiency and drug loading" section.

\section{In vivo Treatment Efficacy of LPNs in Mice}

When the tumor volume of PCa xenograft mice reached about $100 \mathrm{~mm}^{3}$, the mice were randomly divided into eight groups (10 mice per group). ORL/HA-CBZ/LPNs, HACBZ/LPNs, ORL/LPNs, blank LPNs, free ORL/HACBZ, free ORL, free HA-CBZ, and saline solution (0.9\%) (each contained ORL $5 \mathrm{mg} / \mathrm{kg}$ and/or HA-CBZ $10 \mathrm{mg} / \mathrm{kg}$ ) were injected via the tail vein at day $0,3,6$, 9, and $12 \cdot{ }^{39,44}$ From the first day of treatment, tumor volume and body weight were measured every three days. The tumor volume (TV) was measured by calipers and calculated using the equation: TV $(\%)=$ length $\times$ width $^{2} / 2$. Blood was also collected to detect the serum markers including blood urea nitrogen (BUN), lactate dehydrogenase (LDH), alanine aminotransferase (ALT), and white blood cells (WBC). ${ }^{46,47}$

\section{Statistical Analysis}

Data were expressed as mean \pm standard deviations. Statistical analyses were performed using an unpaired, two-tailed Student's $t$-test (between two groups) or oneway analysis of variance (ANOVA) (among three or more groups) followed by Tukey's test. $\mathrm{P}<0.05$ was considered significant.

\section{Results}

\section{Characterization of HA-CBZ and LPNs}

HA-CBZ was characterized by ${ }^{1} \mathrm{H}-\mathrm{NMR}$. The peak at 4.08 ppm presented the formation of the ester bonds (Figure 1). Other peaks belong to HA and CBZ, respectively. The size of the HA contained drugs loaded and blank LPNs were around $150 \mathrm{~nm}$ with narrow PDIs (less than 0.20) (Table 1). However, ORL/LPNs showed a size of $123.1 \pm 5.5 \mathrm{~nm}$, which could prove the presence of HA enlarged the size of the particles. ORL/HA-CBZ/LPNs showed a dim ring surrounding the core, which may be evidence of the HA decoration on the particles' surface (Figure 3A). LPNs also exhibited more negative zeta potentials when HA was applied. The EEs of LPNs were about 90\% with different DLs summarized in Table 1.

\section{Stability in Serum and During Storage}

The stability of LPNs was monitored by measuring the mean diameter of the LPNs during the storage time or in the presence of FBS. During the stability studies, there were no remarkable changes in the particle size in the presence of plasma (Figure 2A) and during 3 months of storage at $4^{\circ} \mathrm{C}$ (Figure 2B).

\section{In vitro Drug Release}

Figure 3B and $\mathrm{C}$ illustrated that LPNs had sustained release behaviors. CBZ showed similar release from ORL/HA-CBZ/LPNs and HA-CBZ/LPNs. On the contrary, ORL release from ORL/HA-CBZ/LPNs and ORL/ LPNs are different. ORL released slower from ORL/HACBZ/LPNs (60 h to complete) than that of ORL/LPNs (36 $\mathrm{h}$ to complete), which may be attributed to the HA coating delayed the drug release.

\section{In vitro Anti-Proliferative Effect}

In vitro anti-proliferative effect of LPNs was evaluated on LNCaP, PC3 and RWPE-2 cells. Both LNCaP and PC3 cells were inhibited in concentration deepened manners

Table I The Particle Size, Zeta Potential, Entrapment Efficiency, and Drug Loading of LPNs

\begin{tabular}{|c|c|c|c|c|c|c|c|}
\hline \multirow[t]{2}{*}{ LPNs } & \multirow[t]{2}{*}{ Particle Size (nm) } & \multirow[t]{2}{*}{ Zeta Potential (mV) } & \multirow[t]{2}{*}{ PDI } & \multicolumn{2}{|c|}{ EE (\%) } & \multicolumn{2}{|c|}{ DL (\%) } \\
\hline & & & & ORL & CBZ & ORL & CBZ \\
\hline Blank LPNs & $122.9 \pm 5.9$ & $-7.3 \pm 0.8$ & $0.13 \pm 0.02$ & I & I & I & l \\
\hline ORL/LPNs & $123.1 \pm 5.5$ & $-6.6 \pm 0.9$ & $0.15 \pm 0.03$ & $89.5 \pm 3.8$ & l & $6.3 \pm 0.5$ & l \\
\hline HA-LPNs & $156.8 \pm 5.9$ & $-19.5 \pm 2.8$ & $0.15 \pm 0.02$ & I & I & I & l \\
\hline HA-CBZ/LPNs & $149.3 \pm 6.1$ & $-17.3 \pm 3.1$ & $0.17 \pm 0.03$ & I & $91.3 \pm 3.4$ & l & $5.9 \pm 0.6$ \\
\hline ORL/HA-CBZ/LPNs & $150.9 \pm 6.4$ & $-18.9 \pm 3.3$ & $0.16 \pm 0.04$ & $90.2 \pm 3.4$ & $88.5 \pm 2.9$ & $5.6 \pm 0.7$ & $5.5 \pm 0.8$ \\
\hline
\end{tabular}

Note: Data presented as means \pm standard deviations. 

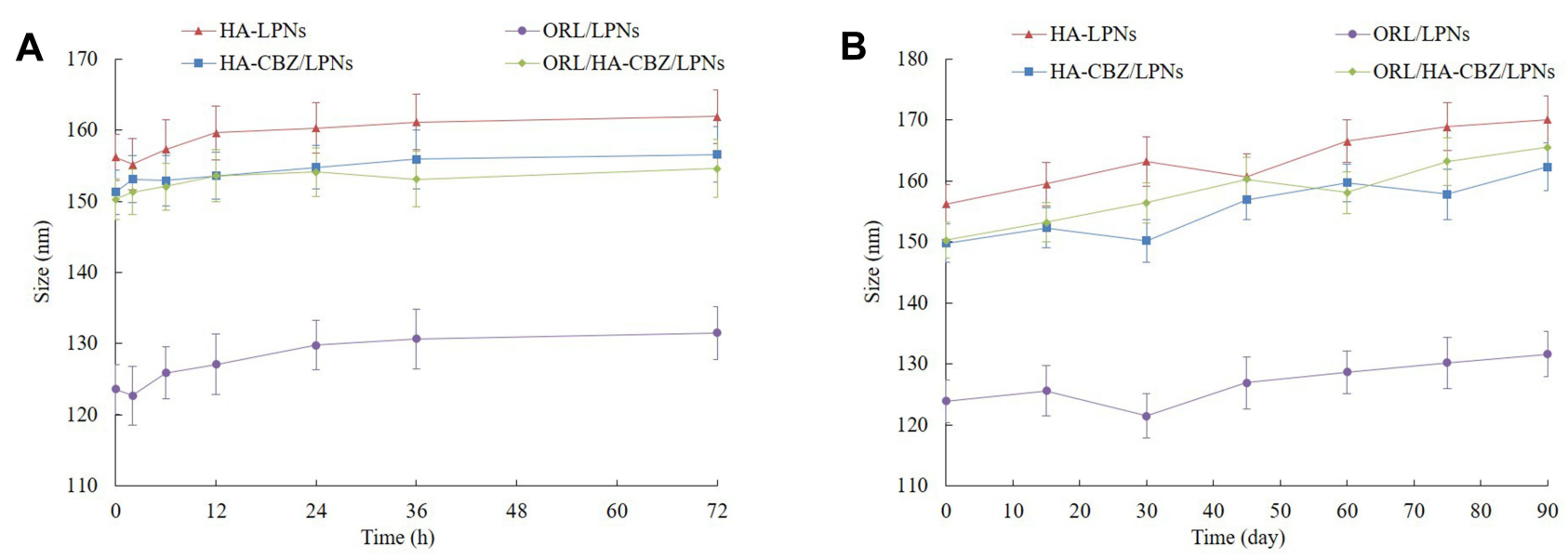

Figure 2 Stability presented as changes in the particle size in the presence of plasma $(\mathbf{A})$ and during 3 months of storage at $4^{\circ} \mathrm{C}(\mathbf{B})$.

A

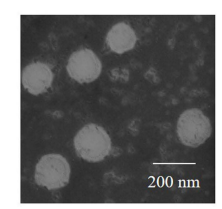

TEM: ORL/HA-CBZ/LPNs
B

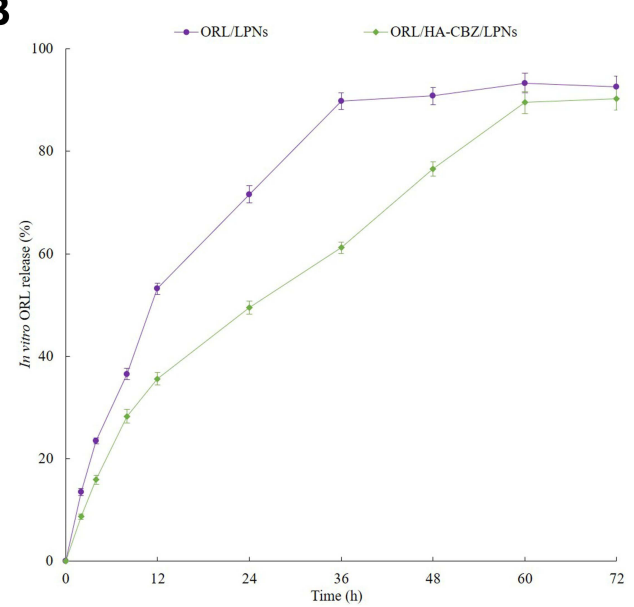

C

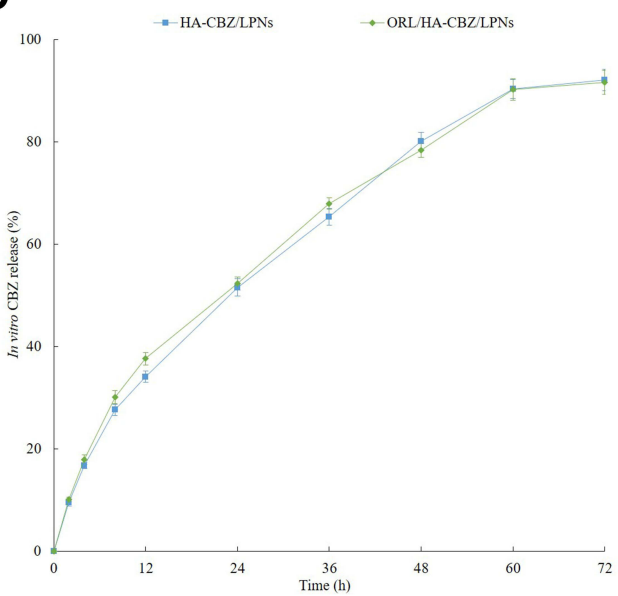

Figure 3 TEM image of ORL/HA-CBZ/LPNs (A). The release profiles of ORL (B) and HA-CBZ (C) from LPNs. Data presented as Means \pm SD.

(Figure 4). ORL/HA-CBZ/LPNs exhibited the most remarkable cell inhibition efficiency (less than $30 \%$ of cell alive at the highest toxicity), showing less cell viability at the end of the study than single drug loaded LPNs and free drugs $(\mathrm{P}<0.05)$. All the drug(s) contained formulas showed considerable anti-proliferative effect compared with control $(\mathrm{P}<0.05)$. Blank LPNs did not show obvious cytotoxicity. Drugs loaded LPNs and free drugs showed similar effects on RWPE-2 cells, which may prove the specific ability of LPNs on cancer cells.

\section{Synergistic Effects Evaluation}

The synergistic effects of ORL and CBZ in one LPNs system were evaluated on the basis of $\mathrm{CI}_{50}$ values of different drug ratios. Table 2 shows that when ORL: HACBZ ratios vary from 5:1 to $1: 5$, synergistic effects were achieved $\left(\mathrm{CI}_{50}<1\right)$. The minimum $\mathrm{CI}_{50}$ value $(0.54)$ was achieved by the ratio of $1: 2$, which was determined as the drug amounts for the ORL/HA-CBZ/LPNs preparation.

\section{Cellular Uptake}

The cellular uptake efficiency of HA contained LPNs and blank LPNs showed different manners (Figure 5). HA contained LPNs (around 69\%) were taken up more by cancer cells than that of blank LPNs $(43.5 \%, \mathrm{P}<0.05)$, which may be explained by the HA coating that improved the uptake of the LPNs.

\section{In vivo Tissue Distribution and Pharmacokinetics}

HA contained ORL/HA-CBZ/LPNs and HA-CBZ/LPNs showed higher drug distribution in tumor than non-HA involved ORL/LPNs $(\mathrm{P}<0.05)$, the latter exhibited higher drug accumulation compared with free ORL/HA-CBZ 

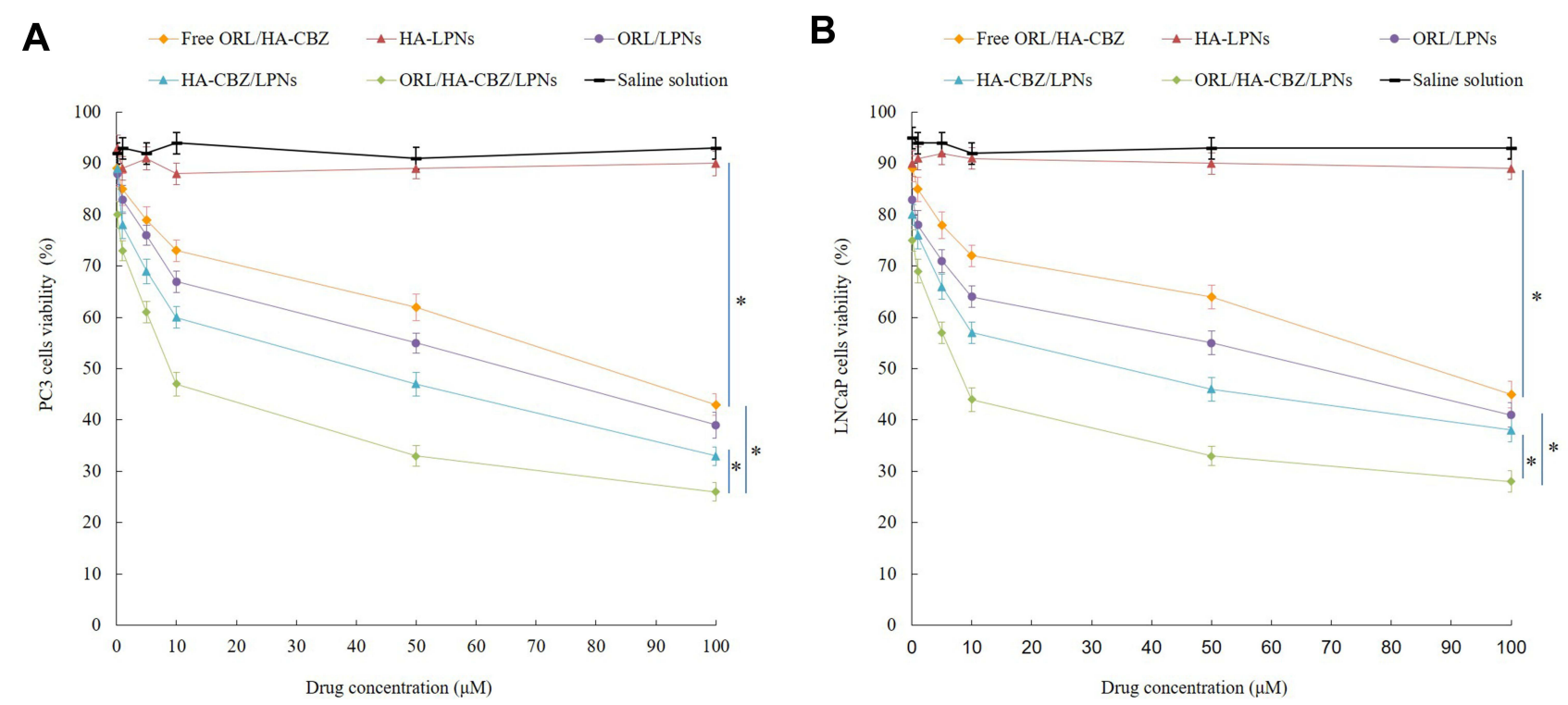

Figure 4 In vitro anti-proliferative effect of LPNs evaluated on LNCaP $(\mathbf{A})$ and PC3 cells $(\mathbf{B})$. Data presented as Means \pm SD. $* \mathrm{P}<0.05$.

$(\mathrm{P}<0.05)$ (Figure 6). To be noticed, the drug accumulation in heart for free ORL/HA-CBZ was higher than that of LPNs formulations $(\mathrm{P}<0.05)$. The pharmacokinetic parameters plasma drug peak concentration $\left(\mathrm{C}_{\max }\right)$, halflife of drugs $\left(\mathrm{T}_{1 / 2}\right)$, and area under the curve (AUC) were summarized in Table 3.

\section{In vivo Treatment Efficacy}

To test the in vivo treatment efficacy of the drugs loaded LPNs and free drugs, PCa xenograft mice were applied. Figure 7A shows that tumor growth was not affected by blank LPNs, with the similar growth curves to the saline solution group. However, the tumor volume was significantly inhibited by the drugs contained groups. When drugs were loaded in LPNs (ORL/HA-CBZ/LPNs), a higher tumor growth inhibition effect was achieved compared with free drugs (free ORL/HA-CBZ) $(\mathrm{P}<$ 0.05). ORL/HA-CBZ/LPNs also illustrated more

Table $2 \mathrm{Cl}_{50}$ and the Corresponding $\mathrm{IC}_{50}$ Values of ORL/HACBZ/LPNs at Different ORL to HA-CBZ Weight Ratio

\begin{tabular}{|l|c|c|c|}
\hline $\begin{array}{l}\text { ORL: HA-CBZ } \\
\text { (w:w) }\end{array}$ & $\begin{array}{c}\text { IC }_{\mathbf{5 0}} \text { of ORL } \\
(\mu \mathrm{M})\end{array}$ & $\begin{array}{c}\text { IC }_{\mathbf{5 0}} \text { of } \mathrm{HA}-\mathrm{CBZ} \\
(\mu \mathrm{M})\end{array}$ & $\mathbf{C l}_{\mathbf{5 0}}$ \\
\hline $5: 1$ & $16.7 \pm 1.9$ & $3.3 \pm 0.6$ & 0.82 \\
2:I & $11.3 \pm 1.1$ & $5.7 \pm 0.9$ & 0.86 \\
$\mathrm{I}: \mathrm{I}$ & $7.6 \pm 1.3$ & $7.7 \pm 1.1$ & 0.90 \\
$\mathrm{I}: 2$ & $2.6 \pm 0.5$ & $5.2 \pm 1.2$ & 0.54 \\
$\mathrm{I}: 5$ & $2.1 \pm 0.3$ & $10.5 \pm 1.8$ & 0.98 \\
\hline
\end{tabular}

Note: Data presented as means \pm standard deviations. pronounced antitumor ability than single drug loaded HACBZ/LPNs and ORL/LPNs $(\mathrm{P}<0.05)$.

\section{In vivo Tolerance Analysis}

To verify the impact of LPNs on systemic toxicity in vivo, the body weight and serum markers of mice were recorded. No significant difference in the mice weights was found among the tested groups, revealing that LPNs did not introduce toxicity. When compared with the saline control group, free drug(s) groups showed increased ALT values, while LPNs groups showed negligible changes of ALT, LDH, BUN, and WBC levels over the control group (Figure 7B and $\mathrm{C}$ ). These results indicated the well tolerance of LPNs at the tested dose, which did not bring about obvious systemic toxicity when administered in vivo.

\section{Discussion}

To achieve the combination therapy of $\mathrm{PCa}$, an HA decorated, CBZ-prodrug and ORL co-loaded LPNs were constructed in this study. First, HA was conjugated with CBZ to produce HA-CBZ. HA-coated Vorinostat-loaded lipid nanoparticles were developed by Tran et al and improved drug delivery to CD44 Overexpressing cancer cells was achieved by selective ability of HA. In our study, LPNs improved the ability of the delivery system and dual drugs could bring the synergistic anticancer effect. ${ }^{45}$

A single-step nanoprecipitation method was employed to prepare LPNs. Liang et al, describe that this method has the following advantages: simplicity, lower energy consumption, narrow size distribution and nice dispersibility 


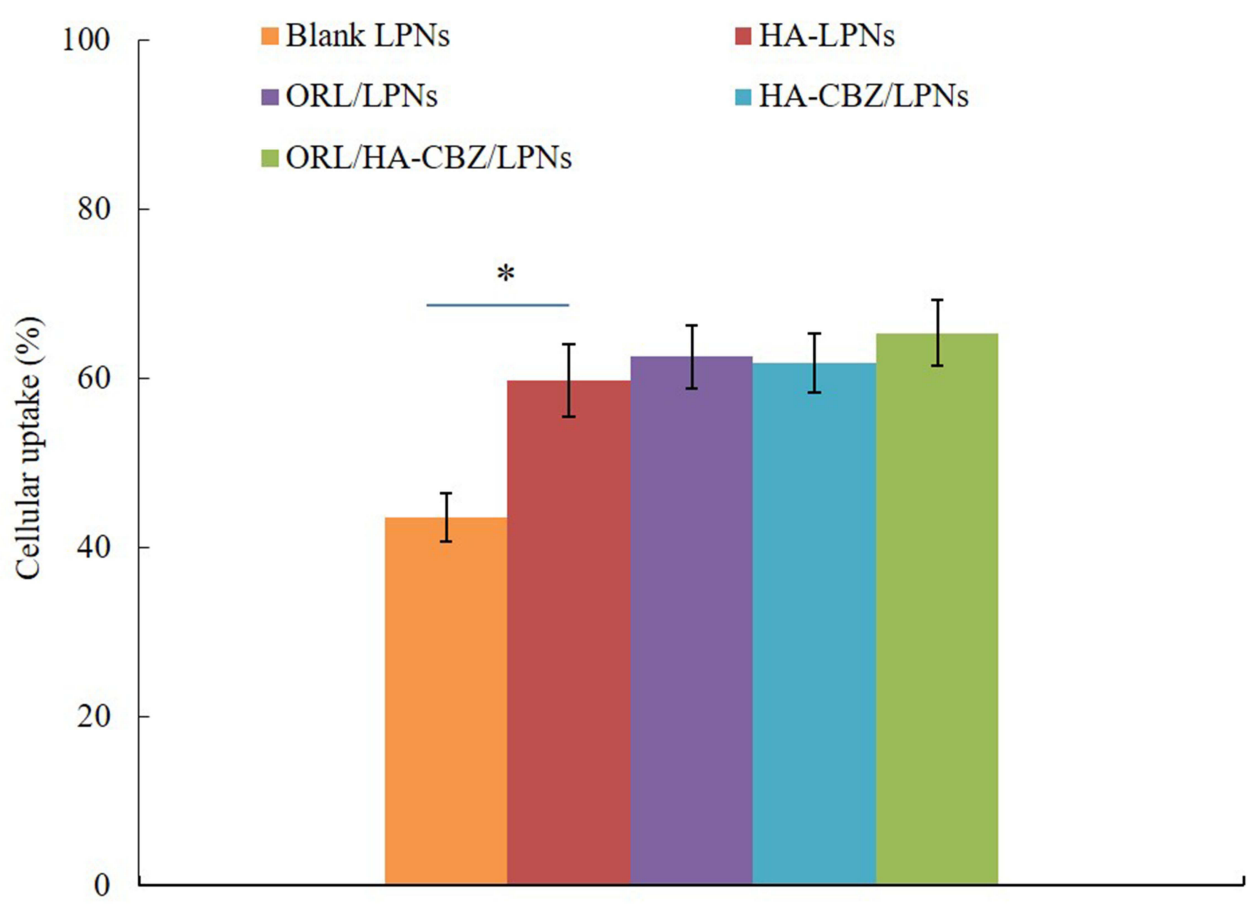

Figure 5 Cellular uptake of LPNs. Data presented as Means \pm SD. $* P<0.05$
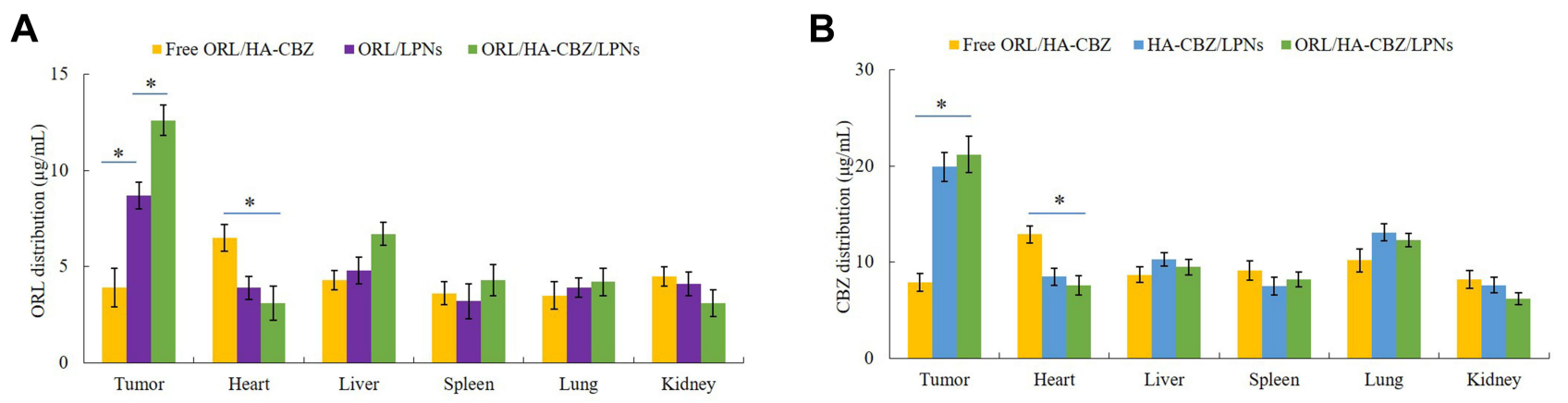

Figure 6 In vivo tissue biodistribution of $\mathrm{ORL}(\mathbf{A})$ and $C B Z(\mathbf{B})$. Data presented as Means $\pm \mathrm{SD}$. $* \mathrm{P}<0.05$.

of the nanoparticles. ${ }^{46}$ They argued that this method facilitates the incorporation of lipophilic drugs into nanoparticles, thus increasing the EE of the delivery systems. EEs

Table 3 The Pharmacokinetic Parameters

\begin{tabular}{|l|c|c|c|}
\hline Parameters & Drugs & $\begin{array}{c}\text { Free ORL/HA- } \\
\text { CBZ }\end{array}$ & $\begin{array}{c}\text { ORL/HA-CBZ } \\
\text { /LPNs }\end{array}$ \\
\hline $\mathrm{C}_{\max }(\mathrm{L} / \mathrm{kg} / \mathrm{h})$ & ORL & $11.6 \pm 0.9$ & $12.9 \pm 1.1$ \\
& HA-CBZ & $18.9 \pm 1.8$ & $20.7 \pm 1.9$ \\
\hline $\mathrm{T}_{1 / 2}(\mathrm{~h})$ & ORL & $0.9 \pm 0.1$ & $3.9 \pm 0.6^{*}$ \\
& HA-CBZ & $1.5 \pm 0.3$ & $5.6 \pm 0.9^{*}$ \\
\hline \multirow{2}{*}{$\mathrm{AUC}(\mathrm{mg} / \mathrm{L} \cdot \mathrm{h})$} & ORL & $31.5 \pm 2.1$ & $129.8 \pm 4.9^{*}$ \\
& HA-CBZ & $58.6 \pm 2.9$ & $198.4 \pm 7.9^{*}$ \\
\hline
\end{tabular}

Notes: Data presented as means \pm standard deviations; $* \mathrm{P}<0.05$ compared with free ORL/HA-CBZ. of LPNs tested in this section were about $90 \%$, which was in accordance with their findings. Centrifugal filters with a weight cut-off of 10,000 Da were used by researchers for purification by removing the free molecules and made narrow polydispersity index, which was also applied in this study. ${ }^{28,47}$ LPNs exhibited more negative zeta potentials when HA was applied, which may reduce the systematic toxicity and were important for efficient cancer therapy. ${ }^{48}$ Yan et al developed a negatively charged, docetaxel and curcumin co-loaded nanoparticles $(-38 \mathrm{mV})$ for prostate cancer therapy. ${ }^{49}$ They argued that the negative surface charge of NPs can reduce the systematic toxicity and were important for efficient cancer therapy. Lu and colleagues also concluded that the negatively charged HA added negative charge to the NPs and obtained a more 

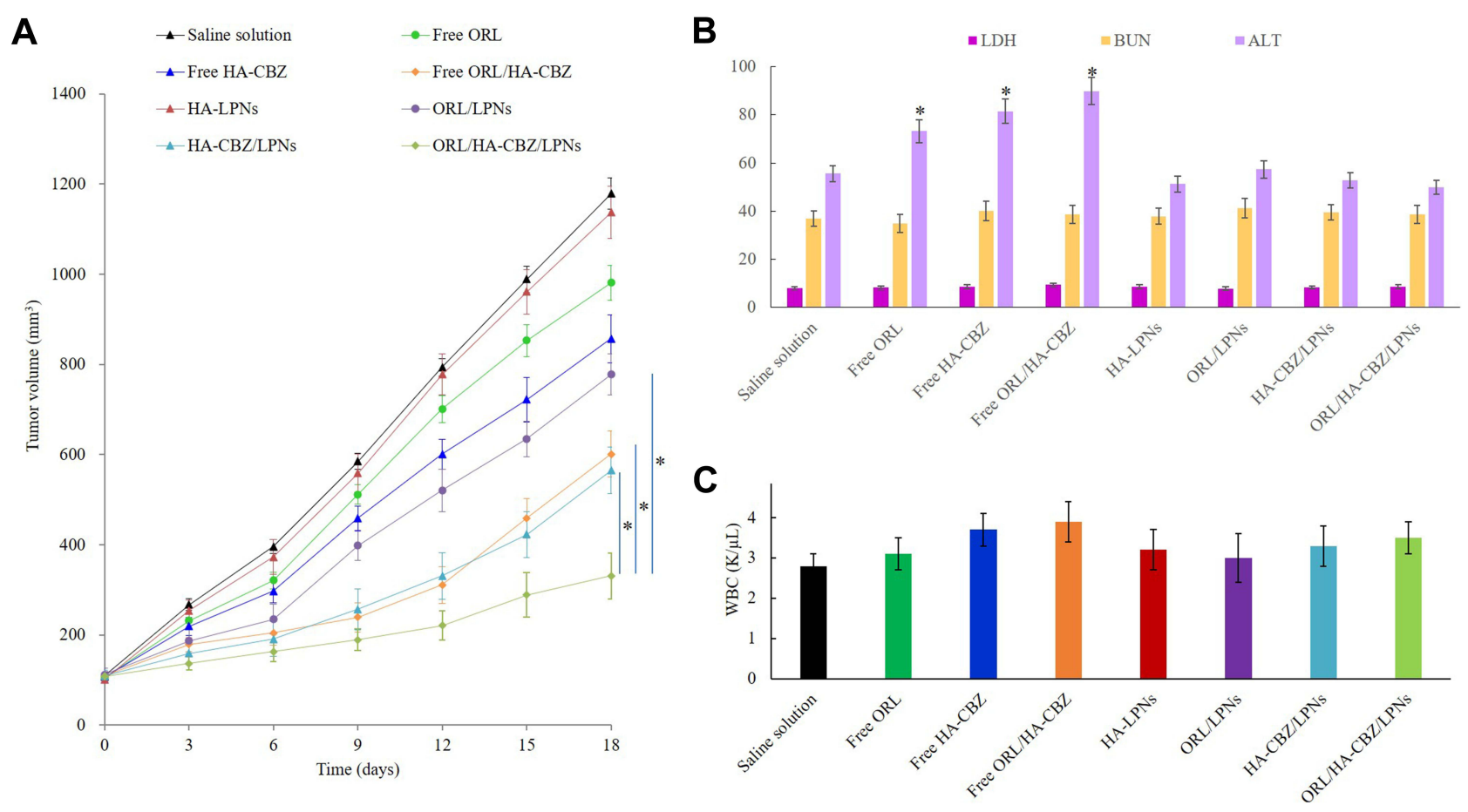

Figure $\mathbf{7}$ In vivo anti-tumor efficacy and toxicity of LPNs evaluated by the curve of tumor volume (A), the serum markers (B), and WBC (C) after treatment. Data presented as Means \pm SD. $* P<0.05$.

negatively charged NPs for the delivery to lung cancer cells. $^{25}$ Gao et al discussed that HA ligands would enhance the cancer cell-specific adherence of the NPs, thus resulting in cancer cells permeation. ${ }^{50}$

The plasma and long-term stability of LPNs would have vital importance when the complexes are administered in vivo. One of the major concerns for nanoparticles is the electrostatic interaction between the vectors and the plasma composition such as proteins, which will increase the size of the nanoparticles. ${ }^{51}$ During the stability studies, there were no remarkable changes in the particle size in the presence of plasma and during 3 months of storage at $4^{\circ} \mathrm{C}$, which would prove the ability of the system when administration in vivo and enhance the targeting proportion of the vector to the target organ, and thus would be favorable to improve the therapeutic effects.

A significant advantage of the nano-system is the sustained or controlled release of the drug due to the enhanced permeability and retention effect (EPR) effects, which greatly enhances the bioavailability and reduces the side effects of the drugs. ${ }^{52}$ The drugs were released from LPNs in sustained behaviors in the study carried out by Shao et al. ${ }^{52}$ They discussed that the dual drugs were mainly located in the polymeric core and the shell needs to be firstly destabilized and then the core may be corroded and let the drugs release slowly. Also, their point of view: "HA modified LPHNs reveal slower release than the nonmodified nanoparticles, which may cause by the HA coating on the LPNs surface which delayed the drug release" is the same as we found in the present research.

In vitro anti-proliferative effect of LPNs was assessed by MTT assays in order to test the inhibitory effect of samples on tumor cell growth. ${ }^{53}$ Higher cytotoxicity of drugs loaded LPNs compared with free drugs indicates that nano-systems can enhance the cytotoxicity. To validate the synergistic effect of drugs co-loaded NPs, the combination index (CI) was further determined using the isobologram equation of Chou and Talalay. ${ }^{54}$ ORL/HA-CBZ/LPNs displayed a minimum $\mathrm{CI}_{50}$ value when ORL-to-HA-CBZ ratio was $1: 2(\mathrm{w} / \mathrm{w})$ varying from $5: 1$ to $1: 5$, which means synergistic effects were achieved and this drug ratio could develop the ability of the drugs to a large extent.

In vivo tissue distribution show that HA LPNs show higher drug distribution in tumor compared with non-HA involved ORL/LPNs, while the latter exhibit more accumulation in tumor than free ORL\&CBZ. This phenomenon could be explained by the target ability of HA and the EPR effect on the tumor site that let the LPNs accumulated easily in the tumor. ${ }^{55}$ ORL/HA-CBZ/LPNs illustrated the most significant in vivo tumor inhibition efficiency, which 
was better than single drug loaded HA-CBZ/LPNs, ORL/ LPNs and free drugs formulations. No significant difference in the mice weights was found among the tested groups, revealing that LPNs did not introduce toxicity. When compared with the saline control group, free $\operatorname{drug}(\mathrm{s})$ groups showed increased ALT values, while LPNs groups showed negligible changes of WBC, ALT, LDH, and BUN levels over the control group. This could be explained by the LPNs would reduce the side effects of drugs. ${ }^{46}$ These results indicated the well tolerance of LPNs at the tested dose, which did not bring about obvious systemic toxicity in vivo when administered in vivo.

\section{Conclusion}

In summary, Cabazitaxel-prodrug was firstly synthesized and ORL/HA-CBZ/LPNs were prepared. ORL/HA-CBZ /LPNs showed remarkable synergism cytotoxicity and the best tumor inhibition efficiency in mice with negligible systemic toxicity. ORL/HA-CBZ/LPNs can be highly useful for targeted prostate cancer therapy.

\section{Disclosure}

The authors have no declaration of interest.

\section{References}

1. Siegel R, DeSantis C, Virgo K, et al. Cancer treatment and survivorship statistics, 2012. CA Cancer J Clin. 2012;62(4):220-241. doi:10.3322/caac.21149

2. Center MM, Jemal A, Lortet-Tieulent J, et al. International variation in prostate cancer incidence and mortality rates. Eur Urol. 2012;61 (6):1079-1092. doi:10.1016/j.eururo.2012.02.054

3. Loblaw DA, Virgo KS, Nam R, et al.; American Society of Clinical Oncology. Initial hormonal management of androgen-sensitive metastatic, recurrent, or progressive prostate cancer: 2006 update of an American Society of Clinical Oncology practice guideline. $J$ Clin Oncol. 2007;25(12):1596-1605. doi:10.1200/JCO.2006.10.1949

4. Hussain M, Tangen CM, Higano C, et al.; Southwest Oncology Group Trial 9346 (INT-0162). Absolute prostate-specific antigen value after androgen deprivation is a strong independent predictor of survival in new metastatic prostate cancer: data from Southwest Oncology Group Trial 9346 (INT-0162). J Clin Oncol. 2006;24(24):3984-3990. doi:10. 1200/JCO.2006.06.4246

5. de Bono JS, Oudard S, Ozguroglu M, et al.; TROPIC Investigators. Prednisone plus cabazitaxel or mitoxantrone for metastatic castration-resistant prostate cancer progressing after docetaxel treatment: a randomised open-label trial. Lancet. 2010;376(9747): 1147-1154. doi:10.1016/S0140-6736(10)61389-X

6. Mellado B, Jimenez N, Marin-Aguilera M, Reig O. Diving into cabazitaxel's mode of action: more than a taxane for the treatment of castration-resistant prostate cancer patients. Clin Genitourin Cancer. 2016;14(4):265-270. doi:10.1016/j.clgc.2015.12.030

7. Fitzpatrick JM, de Wit R. Taxane mechanisms of action: potential implications for treatment sequencing in metastatic castration-resistant prostate cancer. Eur Urol. 2014;65(6):1198-1204. doi:10.1016/j.eururo.2013. 07.022
8. Vrignaud P, Sémiond D, Lejeune P, et al. Preclinical antitumor activity of cabazitaxel, a semisynthetic taxane active in taxane-resistant tumors. Clin Cancer Res. 2013;19(11):2973-2983. doi:10.1158/1078-0432.CCR-12-3146

9. Coors EA, Seybold H, Merk HF, Mahler V. Polysorbate 80 in medical products and nonimmunologic anaphylactoid reactions. Ann Allergy Asthma Immunol. 2005;95(6):593-599. doi:10.1016/S1081-1206(10) 61024-1

10. Fernández $\mathrm{O}$, Afonso J, Vázquez $\mathrm{S}$, et al. Metastatic castration-resistant prostate cancer: changing landscape with cabazitaxel. Anticancer Drugs. 2014;25(3):237-243. doi:10.1097/ CAD.0000000000000045

11. Paller CJ, Antonarakis ES. Cabazitaxel: a novel second-line treatment for metastatic castration-resistant prostate cancer. Drug Des Devel Ther. 2011;5:117-124.

12. Souza C, Pellosi DS, Tedesco AC. Prodrugs for targeted cancer therapy. Expert Rev Anticancer Ther. 2019;19(6):483-502. doi:10.1080/14737140.2019.1615890

13. Shuai Q, Zhao G, Lian X, et al. Self-assembling poly(ethylene glycol)-block-polylactide-cabazitaxel conjugate nanoparticles for anticancer therapy with high efficacy and low in vivo toxicity. Int J Pharm. 2020;574:118879. doi:10.1016/j.ijpharm.20 19.118879

14. Hoang B, Ernsting MJ, Tang WS, et al. Cabazitaxel-conjugated nanoparticles for docetaxel-resistant and bone metastatic prostate cancer. Cancer Lett. 2017;410:169-179. doi:10.1016/j.canlet.2017. 09.029

15. Zhang X, Li X, You Q, Zhang X. Prodrug strategy for cancer cell-specific targeting: a recent overview. Eur J Med Chem. 2017;139:542-563. doi:10.1016/j.ejmech.2017.08.010

16. Huang WY, Lin JN, Hsieh JT, et al. Nanoparticle targeting CD44-positive cancer cells for site-specific drug delivery in prostate cancer therapy. ACS Appl Mater Interfaces. 2016;8(45):307 22-30734. doi:10.1021/acsami.6b10029

17. Souchek JJ, Wojtynek NE, Payne WM, et al. Hyaluronic acid formulation of near infrared fluorophores optimizes surgical imaging in a prostate tumor xenograft. Acta Biomater. 2018;75:323-333. doi:10.1016/j.actbio.2018.06.016

18. Skarmoutsos I, Skarmoutsos A, Katafigiotis I, et al. Hyaluronic acid and hyaluronidase as possible novel urine biomarkers for the diagnosis of prostate cancer. Med Oncol. 2018;35(7):97. doi:10.1007/ s12032-018-1157-9

19. Mahira S, Kommineni N, Husain GM, Khan W. Cabazitaxel and silibinin co-encapsulated cationic liposomes for CD44 targeted delivery: a new insight into nanomedicine based combinational chemotherapy for prostate cancer. Biomed Pharmacother. 2019; 110:803-817. doi:10.1016/j.biopha.2018.11.145

20. Souchek JJ, Davis AL, Hill TK, et al. Combination treatment with orlistat-containing nanoparticles and taxanes is synergistic and enhances microtubule stability in taxane-resistant prostate cancer cells. Mol Cancer Ther. 2017;16(9):1819-1830. doi:10.1158/15357163.MCT-17-0013

21. Menendez JA, Vellon L, Colomer R, Lupu R. Pharmacological and small interference RNA-mediated inhibition of breast cancer-associated fatty acid synthase (oncogenic antigen-519) synergistically enhances Taxol (paclitaxel)-induced cytotoxicity. Int J Cancer. 2005;115(1):19-35. doi:10.1002/ijc.20754

22. Menendez JA, Lupu R, Colomer R. Inhibition of tumor-associated fatty acid synthase hyperactivity induces synergistic chemosensitization of HER $-2 /$ neu-overexpressing human breast cancer cells to docetaxel (taxotere). Breast Cancer Res Treat. 2004;84(2):183-195. doi:10.1023/B:BREA.0000018409.59448.60

23. Meena AS, Sharma A, Kumari R, Mohammad N, Singh SV, Bhat MK. Inherent and acquired resistance to paclitaxel in hepatocellular carcinoma: molecular events involved. PLoS One. 2013;8(4): e61524. doi:10.1371/journal.pone.0061524 
24. Hill TK, Davis AL, Wheeler FB, et al. Development of a self-assembled nanoparticle formulation of orlistat, nano-ORL, with increased cytotoxicity against human tumor cell lines. Mol Pharm. 2016;13(3):720-728. doi:10.1021/acs.molpharmaceut.5b00 447

25. Lu G, Cao L, Zhu C, et al. Improving lung cancer treatment: hyaluronic acid-modified and glutathione-responsive amphiphilic TPGS-doxorubicin prodrug-entrapped nanoparticles. Oncol Rep. 2019;42(1):361-369.

26. Guo S, Zhang Y, Wu Z, et al. Synergistic combination therapy of lung cancer: cetuximab functionalized nanostructured lipid carriers for the co-delivery of paclitaxel and 5-demethylnobiletin. Biomed Pharmacother. 2019;118:109225. doi:10.1016/j.biopha.2019.109 225

27. Liu J, Cheng H, Han L, et al. Synergistic combination therapy of lung cancer using paclitaxel- and triptolide-coloaded lipid-polymer hybrid nanoparticles. Drug Des Devel Ther. 2018;12:3199-3209. doi:10. 2147/DDDT.S172199

28. Wang J, Su G, Yin X, et al. Non-small cell lung cancer-targeted, redox-sensitive lipid-polymer hybrid nanoparticles for the delivery of a second-generation irreversible epidermal growth factor inhibitor-Afatinib: in vitro and in vivo evaluation. Biomed Pharmacother. 2019;120:109493. doi:10.1016/j.biopha.2019.109493

29. Yang B, Wang K, Zhang D, et al. Light-activatable dual-source ROS-responsive prodrug nanoplatform for synergistic chemo-photodynamic therapy. Biomater Sci. 2018;6(11):2965-2975. doi:10.1039/C8BM00899J

30. Zhang L, Chan JM, Gu FX, et al. Self-assembled lipid-polymer hybrid nanoparticles: a delivery platform. ACS Nano. 2008;2 (8):1696-1702. doi:10.1021/nn800275r

31. Roese E, Bunjes H. Drug release studies from lipid nanoparticles in physiological media by a new DSC method. J Control Release. 2017;256:92-100. doi:10.1016/j.jconrel.2017.04.032

32. Wang H, Sun G, Zhang Z, Ou Y. Transcription activator, hyaluronic acid and tocopheryl succinate multi-functionalized novel lipid carriers encapsulating etoposide for lymphoma therapy. Biomed Pharmacother. 2017;91:241-250. doi:10.1016/j.biopha.2017.04. 104

33. Zhang K, Lv S, Li X, et al. Preparation, characterization, and in vivo pharmacokinetics of nanostructured lipid carriers loaded with oleanolic acid and gentiopicrin. Int J Nanomedicine. 2013;8:3227-3239. doi:10.2147/IJN.S45031

34. Kim DH, Kim JY, Kim RM, et al. Orlistat-loaded solid SNEDDS for the enhanced solubility, dissolution, and in vivo performance. Int J Nanomedicine. 2018;13:7095-7106. doi:10.2147/IJN.S181175

35. Sun Y, Zhao Y, Teng S, et al. Folic acid receptor-targeted human serum albumin nanoparticle formulation of cabazitaxel for tumor therapy. Int $J$ Nanomedicine. 2018;14:135-148. doi:10.2147/IJN. S181296

36. Chen Y, Deng Y, Zhu C, Xiang C. Anti prostate cancer therapy: aptamer-functionalized, curcumin and cabazitaxel co-delivered, tumor targeted lipid-polymer hybrid nanoparticles. Biomed Pharmacother. 2020;127:110181. doi:10.1016/j.biopha.2020.110181

37. Li W, Qian L, Lin J, et al. CD44 regulates prostate cancer proliferation, invasion and migration via PDK1 and PFKFB4. Oncotarget. 2017;8(39):65143-65151. doi:10.18632/oncotarget.17821

38. Senbanjo LT, AlJohani H, Majumdar S, Chellaiah MA. Characterization of CD44 intracellular domain interaction with RUNX2 in PC3 human prostate cancer cells. Cell Commun Signal. 2019;17(1):80. doi:10.1186/s12964-019-0395-6

39. Paulmurugan R, Bhethanabotla R, Mishra K, et al. Folate receptor-targeted polymeric micellar nanocarriers for delivery of orlistat as a repurposed drug against triple-negative breast cancer. Mol Cancer Ther. 2016;15(2):221-231. doi:10.1158/1535-7163. MCT-15-0579
40. Chou TC, Talalay P. Quantitative analysis of dose-effect relationships: the combined effects of multiple drugs or enzyme inhibitors. $A d v$ Enzyme Regul. 1984;22:27-55. doi:10.1016/0065-2571(84)90007-4

41. Wang G, Wang Z, Li C, et al. RGD peptide-modified, paclitaxel prodrug-based, dual-drugs loaded, and redox-sensitive lipid-polymer nanoparticles for the enhanced lung cancer therapy. Biomed Pharmacother. 2018;106:275-284. doi:10.1016/j.biopha.2018.06.137

42. Wan Z, Xie F, Wang L, Zhang G, Zhang H. Preparation and evaluation of cabazitaxel-loaded bovine serum albumin nanoparticles for prostate cancer. Int J Nanomedicine. 2020;15:5333-5344. doi:10.21 47/IJN.S258856

43. Fusser M, Øverbye A, Pandya AD, et al. Cabazitaxel-loaded Poly (2-ethylbutyl cyanoacrylate) nanoparticles improve treatment efficacy in a patient derived breast cancer xenograft. $J$ Control Release. 2019;293:183-192. doi:10.1016/j.jconrel.2018.11.029

44. Wu M, Wang Y, Wang Y, et al. Paclitaxel-loaded and A10-3.2 aptamer-targeted poly(lactide-co-glycolic acid) nanobubbles for ultrasound imaging and therapy of prostate cancer. Int $J$ Nanomedicine. 2017;12:5313-5330. doi:10.2147/IJN.S136032

45. Tran TH, Choi JY, Ramasamy T, et al. Hyaluronic acid-coated solid lipid nanoparticles for targeted delivery of vorinostat to CD44 overexpressing cancer cells. Carbohydr Polym. 2014;114:407-415. doi:10.1016/j.carbpol.2014.08.026

46. Liang Z, Li J, Zhu B. Lung cancer combination treatment: evaluation of the synergistic effect of cisplatin prodrug, vinorelbine and retinoic acid when co-encapsulated in a multi-layered nano-platform. Drug Des Devel Ther. 2020;14:4519-4531. doi:10.2147/DDDT.S251749

47. Wang $\mathrm{B}, \mathrm{Hu} \mathrm{W}$, Yan $\mathrm{H}$, et al. Lung cancer chemotherapy using nanoparticles: enhanced target ability of redox-responsive and pH-sensitive cisplatin prodrug and paclitaxel. Biomed Pharmacother. 2021;136:111249. doi:10.1016/j.biopha.2021.111249

48. Yan J, Wang Y, Jia Y, et al. Co-delivery of docetaxel and curcumin prodrug via dual-targeted nanoparticles with synergistic antitumor activity against prostate cancer. Biomed Pharmacother. 2017;88:374-383. doi:10.1016/j.biopha.2016.12.138

49. Li M, Feng S, Xing H, Sun Y. Dexmedetomidine and levobupivacaine co-loaded, transcriptional transactivator peptide modified nanostructured lipid carriers or lipid-polymer hybrid nanoparticles, which performed better for local anesthetic therapy? Drug Deliv. 2020;27(1):1452-1460. doi:10.1080/10717544.2020.1831105

50. Gao Z, Li Z, Yan J, Wang P. Irinotecan and 5-fluorouracil-co-loaded, hyaluronic acid-modified layer-by-layer nanoparticles for targeted gastric carcinoma therapy. Drug Des Devel Ther. 2017;11:2595-2604. doi:10.2147/DDDT.S140797

51. Zhang T, Ma J, Li C, et al. Core-shell lipid polymer nanoparticles for combined chemo and gene therapy of childhood head and neck cancers. Oncol Rep. 2017;37(3):1653-1661. doi:10.3892/or.2017.5365

52. Shao Y, Luo W, Guo Q, Li X, Zhang Q, Li J. In vitro and in vivo effect of hyaluronic acid modified, doxorubicin and gallic acid co-delivered lipid-polymeric hybrid nano-system for leukemia therapy. Drug Des Devel Ther. 2019;13:2043-2055. doi:10.2147/ DDDT.S202818

53. Zhang Y, Zhang P, Zhu T. Ovarian carcinoma biological nanotherapy: comparison of the advantages and drawbacks of lipid, polymeric, and hybrid nanoparticles for cisplatin delivery. Biomed Pharmacother. 2019;109:475-483. doi:10.1016/j.biopha.2018.10.158

54. Zhang R, Ru Y, Gao Y, Li J, Mao S. Layer-by-layer nanoparticles co-loading gemcitabine and platinum (IV) prodrugs for synergistic combination therapy of lung cancer. Drug Des Devel Ther. 2017;11:2631-2642. doi:10.2147/DDDT.S143047

55. Wang J. Combination treatment of cervical cancer using folate-decorated, $\mathrm{pH}$-sensitive, carboplatin and paclitaxel co-loaded lipid-polymer hybrid nanoparticles. Drug Des Devel Ther. 2020;14:823-832. doi:10.2147/DDDT.S235098 


\section{Publish your work in this journal}

Drug Design, Development and Therapy is an international, peerreviewed open-access journal that spans the spectrum of drug design and development through to clinical applications. Clinical outcomes, patient safety, and programs for the development and effective, safe, and sustained use of medicines are a feature of the journal, which has also been accepted for indexing on PubMed Central. The manuscript management system is completely online and includes a very quick and fair peer-review system, which is all easy to use. Visit http://www. dovepress.com/testimonials.php to read real quotes from published authors. 